\title{
Lateral migration patterns toward or away from injection wells for earthquake clusters in Oklahoma
}

\author{
J. A. López-Comino1,2,3,4,*, M. Galis5,6, P. M. Mai4, X. Chen7, D. Stich2,3 \\ 1 Institute of Geosciences, University of Potsdam, D-14476 Potsdam-Golm, Germany \\ 2 Instituto Andaluz de Geofísica, Universidad de Granada, Granada, Spain. \\ 3 Departamento de Física Teórica y del Cosmos, Universidad de Granada, Granada, Spain. \\ 4 KAUST, King Abdullah University of Science and Technology, Thuwal, Saudi Arabia. \\ 5 Faculty of Mathematics, Physics and Informatics, Comenius University in Bratislava, Slovakia \\ 6 Earth Science Institute, Slovak Academy of Sciences, Bratislava, Slovakia \\ 7 School of Geosciences, The University of Oklahoma, Norman, OK, USA \\ *Corresponding author: José Ángel López-Comino (lopezcomino@uni-potsdam.de)
}

\begin{abstract}
(up to 300 words)
Exploring the connections between injection wells and seismic migration patterns is key to understanding processes controlling growth of fluid-injection induced seismicity. Numerous seismic clusters in Oklahoma have been associated with wastewater disposal operations, providing a unique opportunity to investigate migration directions of each cluster with respect to the injection-well locations. This study focuses on new strategies to identify and quantify lateral migration toward or away from the injection wells. First, a comprehensive migration analysis is described to reach a representative migration vector for each seismic cluster. Next, we propose a new approach to define a well vector associated with multiple injection points, taking into account cumulative injected-volume and the injection-rate volume weighting, and considering also the expansion of the diffusion front. Injected fluids can be associated with an earthquake cluster only if they have sufficient time to reach the location of the cluster. Finally, new directivity migration parameters are introduced comparing the direction of migration and well vectors. Our results suggest a relationship between migration patterns and the cluster-well distances, but unclear relationship with injected volume and equivalent magnitudes. At shorter distances (up to $\sim 13 \mathrm{~km}$ ), we observe dominantly migration away from injection wells (particularly for distances shorter than $\sim 5 \mathrm{~km}$ ) that could be attributed to pore-pressure changes. However, we also find an opposite behavior, migration toward the wells, for larger distances, suggesting an increasing influence of poroelastic stress changes. This finding is more stable when considering cumulative injected-volume instead of injection-rate.
\end{abstract}




\section{Introduction}

In the last decade, seismic activity observed in Oklahoma has attracted considerable public attention because the annual rate of earthquakes increased since 2009 due to wastewater injection (Ellsworth, 2013; Weingarten et al., 2015; Hincks et al., 2018). Exploring spatio-temporal relations between injection wells and seismic migration patterns is key to understanding the processes controlling the growth of injection-induced seismicity. Earthquakes tend to migrate away from the fluid source following the diffusion of pore pressure, from which hydraulic diffusivity properties can be modeled (Shapiro et al., 2005). However, plausible lateral migration patterns toward or away from injection wells in large-scale fluid-injection stimulated areas such as Oklahoma remain unclear (Haffener et al., 2018). Yet, if lateral migration patterns exist and can be tied to (controllable) injection processes, important implications for (time-dependent) fluid-induced seismic hazard assessment arise. This will also improve our understanding about the rupture processes involved in fluid-injection operations and may help to define practices to reduce the induced seismicity, which is a main concern for the energy technologies. Here, we investigate such properties through a comprehensive migration analysis with respect to multiple injection wells for earthquakes clusters in Oklahoma.

These clusters are defined by applying clustering techniques that associate seismic events into specific groups (or clusters) with specific spatiotemporal relationships, deciphering also different fault structures (Ester et al., 1996; Wang et al., 2013; Zaliapin and Ben-Zion, 2013; Cheng and Chen, 2018). The identification and characterization of these clusters has been well studied in natural and tectonic contexts revealing interesting event migration features such as, for instance, event triggering due to fluid flow (Vidale and Shearer, 2006; Chen et al., 2012; Passarelli et al., 2018). In addition, seismic clusters can be divided into three main categories according different spatiotemporal patterns and migration behaviors (e.g. Vidale and Shearer, 2006): i) mainshockaftershock sequence, beginning with the largest event and aftershocks exhibiting relatively little spreading with time, ii) earthquake swarms, without clear mainshock triggering but somewhat spatially coherent time evolution of the sequence; and iii) an average behavior among the previous two cases. Some authors propose full linear migration modelling to compute the bestfitting migration directions and velocities (Chen and Shearer, 2011; Chen et al., 2012). They explore differences between these cluster categories using statistical parameters such as the time delay of the largest event $\left(t_{\max }\right)$, skewness of the seismic moment distribution $(\mu)$ or the distance separation between the centers of the first half and second half of each cluster $(d s)$.

Recent efforts for improving the existing earthquake catalogues for Oklahoma identified seismicity clusters distributed over the area due to the activation of hundreds of previously unknown faults (Schoenball and Ellsworth, 2017). Analyzing the spatiotemporal evolution of these clusters reveals that seismicity tends to initiate at shallower depth and migrates deeper along faults as the sequence proceeds (Schoenball and Ellsworth, 2017b; Haffener et al., 2018), suggesting possible influence of pressure propagation from injection. Influence of fluid injection on earthquake rupture propagation has also been identified for the largest induced Oklahoma earthquakes (López-Comino and Cesca, 2018; Lui and Huang, 2019). The $2011 \mathrm{Mw} 5.7$ Prague and $2016 \mathrm{Mw}$ 5.0 Cushing earthquakes ruptured away from the injection wells, whereas the 2016 Mw 5.1 Fairview earthquake ruptured toward the injection. Lui and Huang (2019) attributed the difference in rupture directions to expected pressurization of the fault zone, which relates to the 
distance away from injection zones and total injected volume. These studies suggest that fluid injection has an effect on directional properties of earthquake processes.

Haffener et al., (2018) reported complex lateral migration pattern for individual clusters in Oklahoma based on simplified analysis of along-strike migration. In this study, building upon previous studies, we introduce new techniques and parameters to identify and quantify lateral migration patterns toward or away from injection-well locations, and consider multiple variables such as the cluster-well distance, injected volumes and magnitudes to fully assess their influence on lateral migration behaviors of earthquake clusters.

\section{Dataset for study}

We use a relocated earthquake catalog, recorded between 2010 and 2016, with enhanced spatial resolution, a magnitude of completeness $\left(\mathrm{M}_{c}\right)$ of 2.5, and a minimum magnitude of 2.0 (Chen, 2016). We consider individual clusters with at least 20 events identified by Haffener et al., (2018). This resulted in 60 clusters after aftershocks were removed using the space-time windowing method proposed by Uhrhammer (1986) to avoid the space-time imprint of aftershocks. The injection data used in this study are obtained from Oklahoma Corporation Commission websites with monthly data from 1995 to 2017 with a total number of 876 disposal wells (see Data and Resources). Considering maximum well distances of $50 \mathrm{~km}$, the number of injection wells involved in this study is 836 (Figure 1).

\section{Methods}

We perform a comprehensive migration analysis based on the definition of two representative vectors (Figure 2). First, each cluster is characterized by a so-called migration vector calculated using an enhanced migration technique. However, the high density of injection wells makes it difficult to determine causative disposal wells to specific clusters of seismicity. Therefore, we introduce a new methodology to calculate a so-called well vector associated with multiple injection wells. Finally, lateral migration patterns toward or away from injection wells are analyzed by the definition of the new directivity migration parameter $(\kappa)$ comparing the direction of both representative vectors.

\subsection{Migration vector for earthquake clusters}

In our study, we propose a comprehensive migration analysis based on previous approaches involving different numbers of temporal bins of equal duration spanning the period of the entire sequence (Haffener et al., 2018) (Figure 3). Earthquakes in each cluster are divided into 10 temporal bins (Figures 3.a.1 and 3.b.1). We selected this number of bins $(\mathrm{N})$ to reach a robust temporal resolution than other simplified parameters such as $d_{s}$ (i.e. $\mathrm{N}>2$ ) and taking into account the minimum number of events considered to identify each seismic cluster (i.e. $\mathrm{N}<20$ ). Note that large $\mathrm{N}$ leads to a large cluster division where few (or none) events could be embedded in each bin. A spatial bin point is then calculated by averaging epicentral locations in each bin, delineating the migration line of each cluster (Figures 3.a.2 and 3.b.2). 
Next, we define the migration vector $\left(\overrightarrow{v_{m}}\right)$, as the direction from the 1 st temporal bin point to the averaged location of the remaining spatial bin points. Each cluster is then characterized by the azimuth $\Phi$ and length $r$ of the migration vector (Figure 2). The notation $\Phi_{0}$ and ro indicates that all events in each cluster were used to calculate the azimuth and length (Figures 3.a.2 and 3.b.2). To assess uncertainties associated with the calculation of the migration vector, we applied a bootstrap analysis. For each cluster, we calculated 100 migration vectors, randomly removing $10 \%$ of events in each repetition (Figure S1). The final $\Phi$ and $r$ are then defined from the average locations of the heads and tails of all migration vectors (Figures 3.a.3 and 3.b.3). We define the associated uncertainties as $\varepsilon \Phi=\Delta \Phi / 2$, where $\Delta \Phi$ is the maximum difference of azimuths calculated from the bootstrap analysis, and $\varepsilon r$ as the standard deviation of $r$. Significant changes of $\Phi$ between repetitions indicate that the cluster does not have a prevailing direction of migration (Figure 3b). Therefore, we only consider clusters with $\Delta \Phi<45^{\circ}$ in our further analysis. Based on this criterion, the migration vectors for 24 clusters were excluded (Table 1 and Figure S2).

Individual clusters are divided into two groups, according to their spatial migration behavior: migration and non-migration groups, or here so-called strong or weak migration groups. Some authors obtain a statistical significance $(\mathrm{sm})$ ranging from 0.5 to 1.0 to identify each migration group according a fixed threshold value around $0.8-0.85$ (Chen et al., 2012). Using such criteria, Oklahoma clusters reveal almost a parity division with around $40-50 \%$ clusters belonging to group with strong migration (Haffener et al., 2018). We propose a simple way to quantify this property by calculating the ratio of the length of the migration vector $(r)$ to the maximum length of the cluster $\left(d_{\max }\right)$ (Figure 3.a.4 and 3.b.4):

$$
\chi=\frac{r}{d_{\max }}
$$

The migration coefficient $(\chi)$ increases from 0 (no migration) to 1 (strong migration), reaching the maximum value only in the case of migration from one end of the cluster to the other. Uncertainties for $\chi$-values are calculated using the bootstrap analysis. A similar distribution for $\chi$ is obtained using different bins to calculate the migration vector where a value of 0.2 yields similar results as using $s m$ to establish the separation among different migration groups (Figure 4).

\subsection{Well vector for multiple injection-well locations}

The association of seismic clusters to specific wells is crucial for determining whether clusters migrate toward or away from the fluid-injection point. Multiple injection points and the long history of injection volumes in Oklahoma complicate the individual associations for each cluster. Similar areas in Alberta (Canada) had addressed this issue through spatiotemporal association filters, discarding wells potentially not associated with earthquake clusters based on a set of association criteria, for instance, epicenters of all temporally associated earthquakes must be within $5 \mathrm{~km}$ of the well pad surface location (Schultz et al., 2018). 
Here, we propose a new methodology representing multiple injection wells around each cluster using a well vector $\left(\overrightarrow{v_{w}}\right)$ defined as the vector from the 1 st spatial bin point of the cluster (used previously to define the migration vector) to an injection midpoint. The well vector is also characterized by the azimuth $\Phi_{\mathrm{w}}$ and length $r_{w}$ (Figure 2). The injection midpoint is determined as the weighted centroid of locations of wells, taking into account the spatial and temporal distribution of the volume of injected fluids into individual wells and the expansion of the diffusion front. Injected fluids can be associated with a cluster only if they have sufficient time to reach the location of the cluster. Considering a linear diffusion model, we approximate this time by the diffusion front (Shapiro et al., 2005)

$$
t_{D}=\frac{d^{2}}{4 \pi D}
$$

where $D$ is diffusion coefficient and $d$ the distance between the well and cluster. For the analysis, we use a representative value for the diffusion coefficient for Oklahoma area $(D=1.5 \mathrm{~m} / \mathrm{s}$, Haffener et al., 2018). This corresponds, for example, to a delay time to for the diffusion front of about 18 months for a well that is located at $30 \mathrm{~km}$ from a cluster (Figure S3). To account for the effects of diffusion, a well is associated with a cluster only if the fluid-injection started more than tD ago. Next, at each time instant $t$ of the seismic sequence, the weight of an individual well $j$ is adjusted according to reported cumulative injected volume $V_{j}\left(t-t_{D}\right)$ and the injection rate volume $\Delta V_{j}(t-t D)$. Note that we consider $\Delta V$ as the volume injected each month and therefore $t$ increases in steps of 30 days, consistently with the reporting period of injected volumes. Finally, the individual weights are adjusted to account for the expansion of the diffusion front such as a geometrical spreading effect. Assuming dominantly horizontal diffusion, we consider 2D expansion, which leads to the weights in the forms of $V_{j}\left(t-t_{D}\right) / d_{j}$ and $\Delta V_{j}\left(t-t_{D}\right) / d_{j}$. To avoid singularities, we consider $\mathrm{d}=1 \mathrm{~km}$ for wells with $\mathrm{d}<1 \mathrm{~km}$.

Following this procedure, we obtain one injection midpoint for each considered time instant $t$ and their average location then defines the final injection midpoint based on weights from cumulative injected volume and injection rate volumes, respectively. The procedure for cluster 52 is illustrated in Figure 5 for cumulative-injected volume and in Figure 6 for injection-rate volumes. We also define the associated uncertainties as $\varepsilon \Phi_{\mathrm{w}}=\Delta \Phi_{\mathrm{w}} / 2$, where $\Delta \Phi_{\mathrm{w}}$ is the maximum difference of azimuths of individual well vectors, and $\varepsilon_{\mathrm{rw}}$ is the standard deviation of $r_{w}$. Like for the migration vector, also here cases with $\Delta \Phi_{\mathrm{w}}>45^{\circ}$ are considered unstable. Using this criterion, we found 36 stable cases when using the cumulative injected-volume weighting and 22 when using the injection-rate volume weighting (Table 1 and Figure S2).

\subsection{Directivity migration parameter toward or away from injection wells.}

To summarize our results of the comprehensive migration analysis considering multiple injection wells, we define the direction toward or away from injection wells by a parameter $\kappa$ that represents the angle between the migration vector and the well vector:

$$
\kappa=\angle\left(\overrightarrow{v_{m}}, \overrightarrow{v_{w}}\right)
$$

$\kappa$-values range from $0^{\circ}$ to $180^{\circ}$, with $\kappa$-values closer to $0^{\circ}$ indicating an alignment among the migration vector and the well vector for a migration direction toward the injection wells. $\kappa$ - 
values closer to $180^{\circ}$ indicate the opposite behavior, i.e., migration away from injection wells. We note that the migration vector is also affected by fault geometry as it is most likely oriented along the fault strike. For this reason, we define $\kappa<60^{\circ}$ as migration toward wells and $\kappa>120^{\circ}$ as migration away. For intermediate cases $\left(60^{\circ}<\kappa<120^{\circ}\right)$ the migration and well vectors are close to perpendicular, making these cluster a less appropriate choice to decide whether seismicity migrates toward or away from the wells. A summary of all calculated parameters is shown in the Table $\mathrm{S} 1$.

\section{Results}

Different variables are considered to assess their influence in these lateral migration behaviors toward or away from multiple injection wells. In Figure 7 we compare $\kappa$ as a function of: i) length of the well vector, ii) the total weights assigned to the multiple associated wells based on cumulative injected volumes and injection rate volumes, and iii) the equivalent magnitude (sum of the seismic moments of the events in a cluster expressed as moment magnitude following Hanks and Kanamori, 1979). For this comparison and following analysis, we only consider clusters with strong migration $(\chi>0.2)$ : 25 of 36 clusters for the cumulative volume weighting (Figure 7a) and 14 of 22 clusters for the injection rate volume weighting (Figure $7 \mathrm{~b}$ ).

Average values and their errors are calculated for clusters migrating toward and away from the wells to identify potential lateral migration patterns. Depending on $r_{w}$, larger differences among these average toward (13 and $16 \mathrm{~km})$ or away $(8$ and $10 \mathrm{~km})$ values are observed for both weightings. However, no significant changes occur depending on the weighted volumes and the equivalent magnitude. Additionally, the histograms of $\kappa$-values for cumulative injected-volume weighting indicate that a small majority of clusters $(60 \%)$ documents a migration away from the wells (Figure 7.a.4).

\section{Discussion}

Finding migration patterns for induced seismic events by fluid injection can help to understand preferred earthquake rupture processes. For this goal numerous cases under similar geological conditions and fluid-injection histories must be jointly considered to obtain robust results. However, each study area separately shows specific characteristics such as different geometries and (pre-) stress conditions of nearby known/unknown faults around the injection wells where many variables are involved. In this context, induced seismicity in Oklahoma has been well studied by many authors (e.g. Pollyea et al., 2018; Hinks et al., 2018) and seems the most appropriate region showing a significant number of seismic clusters under similar conditions to find plausible lateral migration patterns toward or away from the injection wells.

Our study proposes new strategies to analyze the main variables involved in the identification and characterization of representative migration and well vectors and the estimation of their associated uncertainties. For instance, the considered number of events in each cluster and their locations uncertainties can alter the migration behavior; and the whole histories of injection 
volumes from multiple wells must be considered to define representative injection midpoints for each case. We applied a bootstrap analysis to discard unstable migration vectors and take into account the azimuthal variation of the well vector along the total duration of each seismic cluster reaching stable vectors for $60 \%$ and $36 \%$ of the total clusters depending on the cumulativeinjected volume weighting and injection-rate volume weighting, respectively. In addition, we must consider only clusters with strong migration resulting in $42 \%$ and $23 \%$ of the total cluster depending on the weighting scheme. Despite this significant reduction of the total cluster investigated and these complex conditions where too many parameters may influence the final results, we find a sufficient set of stable cases to analyze this challenging task.

A representative migration line, obtained by joining spatiotemporal bin points identified for a cluster, yields complex shapes/patterns for clusters with no predominant migration direction or with bilateral migration. To compare results for a large number of clusters, we approximate the trajectory by the migration vector. The migration starting point is a relevant parameter to obtain a representative migration vector. It is usually assumed to be the epicenter of the first recorded seismic event in the cluster However, we consider that the migration starts at the 1 st spatial bin point, which more accurately defines the first activated fault area than the location of just the single seismic event. Because the complete spatiotemporal history of the seismic sequence must contribute to defining the head of the migration vector, the average epicentral location of the remaining spatial bin points is used.

We introduced a simple way to quantify the strong/weak migration through a migration coefficient $\chi$, computed from the length of the migration vector and the total length of the cluster. $\chi$-values for all clusters show an asymmetric (positively skewed) distribution with a long tail toward the largest $\chi$-values (Figure 4$)$. A median value of this distribution $(\chi \sim 0.2)$ provides similar results as using $s m$ criterions (Haffener et al., 2018) to divide the Oklahoma clusters into strong/weak migration groups. Clusters with $\chi$-values larger than 0.2 show an observable migration, but below this value the length of the migration vector is too short to observe any predominant migration directions.

In addition, a new strategy weighting considering is proposed to define representative well vectors associated with multiple injection points. The cumulative volume weighting may better represent cumulative effects of pore pressure build up from the beginning of the injection, while injection-rate volume weighting may better represent effect of pore pressure variations. From an operational point of view, injection rate weighting may vary significantly over a short time scale, which can cause significant changes of the direction of the well vector during the course of the cluster (Figure 6b), as documented by 22 identified unstable well vectors. In contrast, the cumulative volume weighting provides more stable results with no unstable well vectors (Table 1). Also, different directions of the well vector can be found for each weighting in the same cluster (Figures 5b and 6b). However, the observed patterns remain stable for different choices of the diffusion coefficient ( $\mathrm{D}=1.25-1.75 \mathrm{~m} 2 / \mathrm{s}$; Figures $8, \mathrm{~S} 4$ and $\mathrm{S} 5$ ), which further supports robustness of the well vector-based approach. 
Regardless of the influence of the weighting on well vector orientation, we observe similar patterns when comparing propagation towards (small $\kappa$ ) and away (large $\kappa$ ) from the wells, depending on $r_{w}$ (Figures 8.a.1 and 8.b.1) and the equivalent magnitude (Figures 8.a.3 and 8.b.3). Significant differences are observed only according to $r_{w}$, suggesting the cluster-well distances as a key factor to control these processes. Both distributions overlap, indicating that there is no monocausal relationship with distance, but the general trend is clear. Accordingly, at shorter cluster-well distances where hydraulic connections between faults and injection wells can be involved, the seismicity is triggered by propagating pore-pressure front (Shapiro et al., 2005). The cumulative injected volume weighting provides the most stable results revealing clearly this pattern for cluster with $\mathrm{r}_{\mathrm{w}}<5 \mathrm{~km}$ (Figure 7.a.1). For larger distances, the previous assumption becomes questionable. Outside of the high-pressure zone, poroelastically-induced Coulombstress-changes should surpass pore pressure changes, providing a plausible triggering mechanism in the far-field of injection wells (Goebel et al., 2017). The transition from pore-pressure dominance to poroelastic stress based on distance could explain the changes in migration pattern at further distances, which is observed for $r_{w}$ in the range between $5-20 \mathrm{~km}$ (Figures 7.a.1 and 7.a.2). For $r_{w}>20 \mathrm{~km}$, our only observation corresponds to the Woodward cluster where poroelastic stress changes significantly exceed pore pressure changes (Goebel et al., 2017), lending support to this interpretation.

On the other hand, other previous works analyzed the preferred rupture directions for the largest Oklahoma earthquakes (López-Comino and Cesca, 2018; Lui and Huang, 2019; Wu et al., 2019). Therefore, we could also compare the migration direction of the clusters with the observed rupture directivity of some specific events such as Prague, Guthrie, Cushing and Fairview earthquakes (Pawnee earthquake is excluded from our cluster dataset after the aftershock removal, Haffener et al., 2018). Unstable migration vectors are identified for Cushing and Fairview seismic sequence while Prague and Guthrie reach stable results (Table 2 and Figure S6). However, these cases do not show strong migration according our definition of $\chi$ and they are not considered to identify migration patterns toward or away from the injection wells. We also note that multiple subevents have been observed in Guthrie (Wu et al., 2019) and Fairview (López-Comino and Cesca, 2018) earthquakes, making rupture directivity difficult to detect.

(Maybe we can remove the next paragraph, please let me know your opinion)

Moreover, Folesky et al. (2016) analyzed the directivity effects of the largest seismic events associated with the stimulation of geothermal reservoir in Basel (Switzerland) and found that the preferred rupture propagation depends on magnitude. They found that events with $\mathrm{ML}_{\mathrm{L}}>\sim 2$ propagated backward into the perturbed volume while smaller events propagated away from the well. Our analysis, with minimum equivalent magnitudes around 3.5, shows a different situation, with a significant number of clusters migrating away from the injection wells, and no clear dependence on magnitude.

\section{Conclusions}

A comprehensive migration analysis is applied to decipher the potential relationship between direction of lateral earthquake migration of induced seismic events and the location of multiple 
injection wells. Representative migration vectors are obtained for each seismic cluster and classified in strong/weak migration through the new migration coefficient $\chi$. We also propose new strategies to define representative well vectors associated with multiple injection points surrounding a seismic cluster considering two types of weighting (cumulative-injected volume and injection-rate volume). Finally, the new directivity migration parameter $\kappa$ is introduced to quantify the direction of lateral migration toward or away from the injection point based on the angle between the migration vector and the well vector. This parameter facilitates the identification of these lateral migration patterns and it can be used to compare results in other fluid-injection stimulated areas.

Albeit the main migration pattern in Oklahoma reflects a downward migration from the Arbuckle layer to the basement (Schoenball and Ellsworth, 2017b, Haffener et al., 2018), we found lateral migration patterns involving the cluster-well distance as the main factor to control preferred migration directions toward or away from the injection wells. Migration away from injection wells is found for distances shorter than $5-13 \mathrm{~km}$, while an opposite migration towards the wells is observed for larger distances, suggesting an increasing influence of poroelastic stress changes. Our findings are more stable when considering cumulative injected volume instead of injection rate. We do not observe any relationship between migration direction and injected volume or equivalent magnitudes.

\section{Data and Resources}

The catalogs for earthquake are obtained from OGS (https://www.ou.edu/ogs) and the seismic clusters from Haffener et al., (2018). The disposal well data are obtained from Oklahoma Corporation Commission (OCC). Methodology was developed and implemented in Python language (available at http://www.python.org) and figures was created using Matplotlib (Hunter, 2007). The supplemental material includes 6 figures and 1 table to go along with the main article to help with understanding the context of the methods and results. 


\section{Acknowledgments}

We thank the Oklahoma Geological Survey (OGS) and USGS for continuous monitoring earthquake activities in Oklahoma. The research presented in this article is supported by King Abdullah University of Science and Technology (KAUST) in Thuwal, Saudi Arabia, by FRAGEN project (Fracture activation in geo-reservoirs - physics of (induced) earthquakes in complex fault networks), URF/1/3389-01-01, BAS/1/1339-01-01, and Spanish project CGL2015-67130-C2-2-R. J.A.L.C has also received funding from the European Union's Horizon 2020 research and innovation programme under the Marie Skłodowska-Curie grant agreement $\mathrm{N}^{\mathrm{o}} 754446$ and UGR Research and Knowledge Transfer Found - Athenea3i; and by the Deutsche Forschungsgemeinschaft (DFG, German Research Foundation) - Projektnummer (407141557). We also thank Justin Rubinstein and Simone Cesca for constructive comments and discussions.

\section{References}

Chen, C. (2016). Comprehensive analysis of Oklahoma earthquakes: From earthquake monitoring to 3D tomography and relocation (Phd dissertation), University of Oklahoma.

Chen, X., P. M. Shearer and R. Abercrombie (2012). Spatial migration of earthquakes within seismic clusters in Southern California: Evidence for fluid diffusion. J. Geophys. Res. 117, B04301.

Cheng, Y. and X. Chen (2018). Characteristics of seismicity inside and outside the Salton Sea Geothermal Field. Bull. Seismol. Soc. Am. 108, 1877-1888.

Ellsworth, W. L. (2013). Injection-induced earthquakes. Science 341, no. 6142, 1-7.

Ester, M., H.-P. Kriegel, J. Sander and X. Xu (1996). A density-based algorithm for discovering clusters in large spatial databases with noise. In Proceedings of the 2nd International Conference on Knowledge Discovery and Data Mining (KDD-96). Portland, OR: AAAI Press.

Goebel, T., M. Weingarten, X. Chen, J. Haffener and E. E. Brodsky (2017). The 2016 Mw 5.1 Fairview, Oklahoma earthquakes: Evidence for long-range poroelastic triggering at $>40$ km from fluid disposal wells. Earth and Planetary Science Letters 472, 50-61.

Folesky, J., J. Kummerow, S. A. Shapiro, M. Häring, and H. Asanuma (2016). Rupture directivity of fluid-induced microseismic events: Observations from an enhanced geothermal system, J. Geophys. Res. 121, no. 11, 8034-8047.

Haffener, J., X. Chen and K. Murray (2018). Multiscale analysis of spatiotemporal relationship between injection and seismicity in Oklahoma. Journal of Geophysical Research: Solid Earth 123, 8711-8731.

Hanks, T. and H. Kanamori (1979). A moment magnitude scale, J. Geophys. Res. 84, 23482350 . 
Hincks, T., W. Aspinall, R. Cooke and T. Gernon (2018). Oklahoma's induced seismicity strongly linked to wastewater injection depth, Science 359 (6381), 1251-1255.

Hunter, J.D. (2007). Matplotlib: A 2D Graphics Environment, Computing in Science \& Engineering 9, 90-95.

López-Comino, J. A. and S. Cesca (2018). Source complexity of an injection induced event: The 2016 Mw 5.1 Fairview, Oklahoma earthquake. Geophys. Res. Lett. 45, 4025-4032.

Lui, S. K. and Y. Huang (2019). Do injection-induced earthquakes rupture away from injection wells due to fluid pressure change? Bull. Seismol. Soc. Am. 109 (1), 358-371.

Passarelli, L., E. Rivalta, S. Jónsson, M. Hensch, S. Metzger, S. S. Jakobsdóttir, F. Maccaferri, F. Corbi and T. Dahm (2018): Scaling and spatial complementarity of tectonic earthquake swarms, Earth and Planetary Science Letters 482, 62-70.

Pollyea, R. M., N. Mohammadi, J. E. Taylor and M. C. Chapman (2018). Geospatial analysis of Oklahoma (USA) earthquakes (2011-2016): Quantifying the limits of regional-scale earthquake mitigation measures. Geology, 46(3), 215-218.

Schoenball, M. and W. L. Ellsworth (2017). Waveform relocated earthquake catalog for Oklahoma and Southern Kansas illuminates the Regional Fault Network. Seismol. Res. Lett. 88(5), 1252-1258.

Schoenball, M., and W. L. Ellsworth (2017b). A systematic assessment of the spatiotemporal evolution of fault activation through induced seismicity in Oklahoma and southern Kansas. Journal of Geophysical Research: Solid Earth 122, 10,189-10,206.

Schultz, R., G. Atkinson, D. W. Eaton, Y. J. Gu and H. Kao (2018). Hydraulic fracturing volume is associated with induced earthquake productivity in the Duvernay play, Science 359, 6373, 304-308.

Shapiro, S. A., S. Rentsch and E. Rothert (2005). Characterization of hydraulic properties of rocks using probability of fluid-induced microearthquakes. Geophysics $\mathbf{7 0}$ (2), F27-F33.

Uhrhammer, R. (1986). Characteristics of northern and southern California seismicity. Earthquake Notes 57 (1), 21.

Vidale, J. E. and P. M. Shearer (2006). A survey of 71 earthquake bursts across southern California: Exploring the role of pore fluid pressure fluctuations and aseismic slip as drivers, J. Geophys. Res. 111, B05312.

Wang, Y., G. Ouillon, J. Woessner, D. Sornette and S. Husen (2013). Automatic reconstruction of fault networks from seismicity catalogs including location uncertainty, Journal of Geophysical Research: Solid Earth 118 (11), 5956-5975.

Weingarten, M., S. Ge, J. Godt, B. Bekins and J. Rubinstein (2015). High-rate injection is associated with the increase in U.S. mid-continent seismicity, Science, 348(6241), 13361340.

Wu, Q., Chen, X., \& Abercrombie, R. E. (2019). Source complexity of the $2015 \mathrm{Mw} 4.0$ Guthrie, Oklahoma earthquake. Geophys. Res. Lett., 46, 4674-4684.

Zaliapin, I. and Y. Ben-Zion (2013). Earthquake clusters in southern California I: Identification and stability.,Journal of Geophysical Research: Solid Earth 118, 2847-2864. 


\section{Full mailing address for each author}

- José Ángel López-Comino (lopezcomino@uni-potsdam.de)

- Martin Galis (martin.galis@uniba.sk)

- Paul Martin Mai (martin.mai@kaust.edu.sa)

- Xiaowei Chen (xiaowei.chen@ou.edu)

- Daniel Stich (stich@ugr.es)

\section{Table and Figure Captions}

Table 1. Total seismic cluster according the stability of migration and well vectors based on different weighting approaches.

Table 2. Azimuths of the migration vector $(\phi)$ and migration coefficients $(\chi)$ for the most important earthquake clusters in Oklahoma analyzed in this study.

Figure 1. Map of wells (inverted triangles) within a radius of $50 \mathrm{~km}$ from the average location in each cluster and 60 seismic clusters in Oklahoma (black dots) detected by a nearest-neighbor approach after aftershocks were removed using a space-time windowing method (Haffener et al., 2018). Wells are scaled (color and size) according to the total injected volume between 1995 2017. Two selected clusters (17 and 52) analyzed in Figure 3 are indicated.

Figure 2. Sketch showing the parameters for calculating the migration and well vector for the comprehensive migration analysis with respect to multiple wells.

Figure 3. Migration analysis for cluster 52 showing results for a stable migration vector (a) and cluster 17 for an unstable migration vector (b). (a.1, b.1) Temporal evolution of the seismic sequence from tini; the color scale indicates association of seismic events with temporal bins. (a.2, b.2) Migration vector calculation; $r_{0}$ and $\Phi_{0}$ represent the length and azimuth of the migration vector calculated using all events in the cluster. (a.3, b.3) Bootstrap analysis to calculate the final length $r$ and azimuth $\Phi$ of the migration vector and their uncertainties. Small white triangles and small white squares depict the heads and tails of 100 migration vectors for the bootstrap analysis. The final migration vector is depicted by a black line from the tail (large white square) to the head (large white triangle). (a.4, b.4) The maximum cluster length ( $\left.d_{\max }\right)$ and the migration coefficient $(\chi)$ are shown with the uncertainties obtained from the bootstrap analysis. $d_{\max }$ (gray line), is defined by the two seismic events farthest from each other (open gray squares).

Figure 4. Histogram for the migration coefficient $(\chi)$ for earthquakes clusters in Oklahoma. The dashed black lines separates weak and strong migration clusters. 
Figure 5. Calculating the well vector for cluster 52 considering the cumulative-injected volume weighting and a diffusion coefficient of $1.5 \mathrm{~m} 2 / \mathrm{s}$. a) Situation at the final time of the seismic sequence tmax. The well vector (black line) is defined from the tail of the migration vector (white square defined in Figure a.3) to the injection midpoint (black square). Length $\left(r_{w}\right)$ and azimuth $\left(\Phi_{\mathrm{w}}\right)$ of the well vector are indicated in the figure header. Wells (inverted triangles) are scaled (color and size) according $\mathrm{V}\left(\mathrm{t}_{\max }-\mathrm{tD}_{\mathrm{D}}\right) / \mathrm{d}$. b) Location of injection midpoints during the seismic sequence (color-coded squares). The final injection midpoint is shown with an open black circle, the final well vector by the black line. Wells are scaled in size as in a). Only the dashed rectangle from a) is shown. c) Cumulative injected volume for the wells associated with the cluster (blue lines) and cumulative seismic moment for the seismic sequence (black line; the largest event is indicated by the star). Red lines indicate the volume that did not affect the cluster due to diffusion constraints, cyan lines indicate data available after the end of seismic sequence.

Figure 6. Calculating the well vector for cluster 52 considering the injection-rate volume weighting and a diffusion coefficient of $1.5 \mathrm{~m} 2 / \mathrm{s}$. a) Situation at the final time of the seismic sequence tmax. The well vector (black line) is defined from the tail of the migration vector (white square defined in Figure a.3) to the injection midpoint (black square). Length $\left(r_{w}\right)$ and azimuth $\left(\Phi_{\mathrm{w}}\right)$ of the well vector are indicated in the figure header. Wells (inverted triangles) are scaled (color and size) according $\Delta \mathrm{V}\left(\mathrm{t}_{\max }-\mathrm{t} \mathrm{D}\right) / \mathrm{d}$. b) Location of injection midpoints during the seismic sequence (color-coded squares). The final injection midpoint is shown with an open black circle, the final well vector by the black line. Wells are scaled in size as in a). Only the dashed rectangle from a) is shown. c) Injection rate volumes for five wells associated with the cluster (blue lines) and cumulative seismic moment for the seismic sequence (black line; the largest event is indicated by the star). Red lines indicate the volume that did not affect the cluster due to diffusion constraints, cyan lines indicate data available after the end of seismic sequence. For each well the injection rate volume $(\Delta \mathrm{V})$ with respect to $t_{\max }$ (open black circle) and the well distance (d) are indicated.

Figure 7. Lateral migration patterns toward or away from injection wells characterized by $\kappa$ values for a diffusion coefficient of $1.5 \mathrm{~m} 2 / \mathrm{s}$. $\kappa$-values for strong migration clusters $(\chi>0.2)$ are plotted as circles scaled in color according the migration coefficient $(\chi)$, considering the cumulative-injected volume weighting (a) and the injection-rate volume weighting (b). Results are shown for each cluster according to the length of the well vector (a.1 and b.1), the total weights assigned to the multiple associated wells in relation to cumulative injected volumes (a.2) and injection rate volumes (b.2) and the equivalent magnitude (a.3 and b.3). Average values and error bars (black squares and lines) are indicated for propagation toward ( $\kappa<60 \square)$ and away $(\kappa$ $>120 \square$ ) from the injection point (see labels). Histograms are also shown including percentages values (a.4 and b.4). Intermediate cases $(60 \square<\kappa<120 \square)$ are not considered (gray background separated by black dashed lines).

Figure 8. Uncertainties of lateral migration patterns toward or away from injection wells assessed for different diffusion coefficients (D) indicated in the y-axis, considering the cumulative-injected volume weighting (a) and the injection-rate volume weighting (b). Results are shown according to the length of the well vector (a.1 and b.1), the total weights assigned to the multiple associated wells in relation to cumulative injected volumes (a.2) and injection rate 
volumes (b.2), and the equivalent magnitude (a.3 and b.3). A gray band, as in Figure 7, is shown in each plot to separate both resulting sets (toward and away). Results from Figure 7 for $D=1.5$ $\mathrm{m} 2 / \mathrm{s}$ are indicated in green color. 


\begin{tabular}{|c|c|c|c|c|}
\cline { 3 - 5 } \multicolumn{1}{c|}{} & $\begin{array}{c}\text { Unstable } \\
\text { migration vector }\end{array}$ & $\begin{array}{c}\text { Unstable } \\
\text { well vector }\end{array}$ & $\begin{array}{c}\text { Unstable } \\
\text { migration \& } \\
\text { well vectors }\end{array}$ & $\begin{array}{c}\text { Stable } \\
\text { migration \& } \\
\text { well vectors }\end{array}$ \\
\hline $\begin{array}{c}\text { Cumulative-injected volume } \\
\text { weighting }\end{array}$ & \multirow{2}{*}{24} & 0 & 0 & 36 \\
\cline { 3 - 5 } $\begin{array}{c}\text { Injection-rate volume } \\
\text { weighting }\end{array}$ & 22 & 8 & 22 \\
\hline
\end{tabular}

Table 1. Total seismic cluster according the stability of migration and well vectors based on different weighting approaches.

\begin{tabular}{|c|c|c|}
\hline Cluster name & $\left.\boldsymbol{\varphi} \mathbf{(}^{\circ}\right)$ & $\begin{array}{c}\text { migration } \\
\text { coefficient }(\chi)\end{array}$ \\
\hline Prague & $22 \pm 19$ & $0.08 \pm 0.01$ \\
\hline Guthrie & $287 \pm 11$ & $0.14 \pm 0.02$ \\
\hline Cushing & $254 \pm 84$ & $0.13 \pm 0.05$ \\
\hline Woodward & $64 \pm 10$ & $0.22 \pm 0.03$ \\
\hline Fairview & $268 \pm 23$ & $0.13 \pm 0.02$ \\
\hline
\end{tabular}

Table 2. Azimuths of the migration vector $(\phi)$ and migration coefficients $(\chi)$ for the most important earthquake clusters in Oklahoma analyzed in this study. 


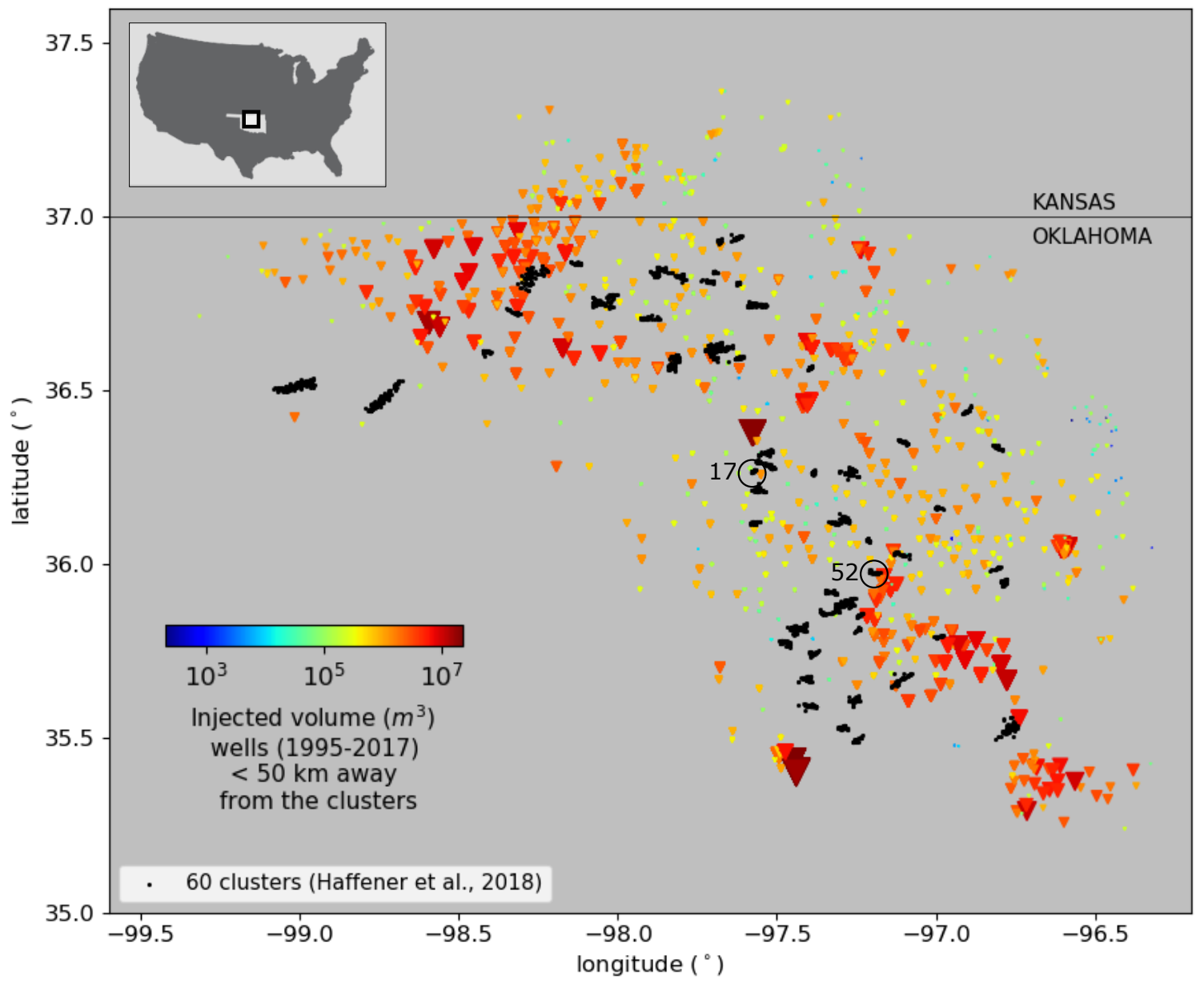

Figure 1. Map of wells (inverted triangles) within a radius of $50 \mathrm{~km}$ from the average location in each cluster and 60 seismic clusters in Oklahoma (black dots) detected by a nearest-neighbor approach after aftershocks were removed using a space-time windowing method (Haffener et al., 2018). Wells are scaled (color and size) according to the total injected volume between 1995 2017. Two selected clusters (17 and 52) analyzed in Figure 3 are indicated. 


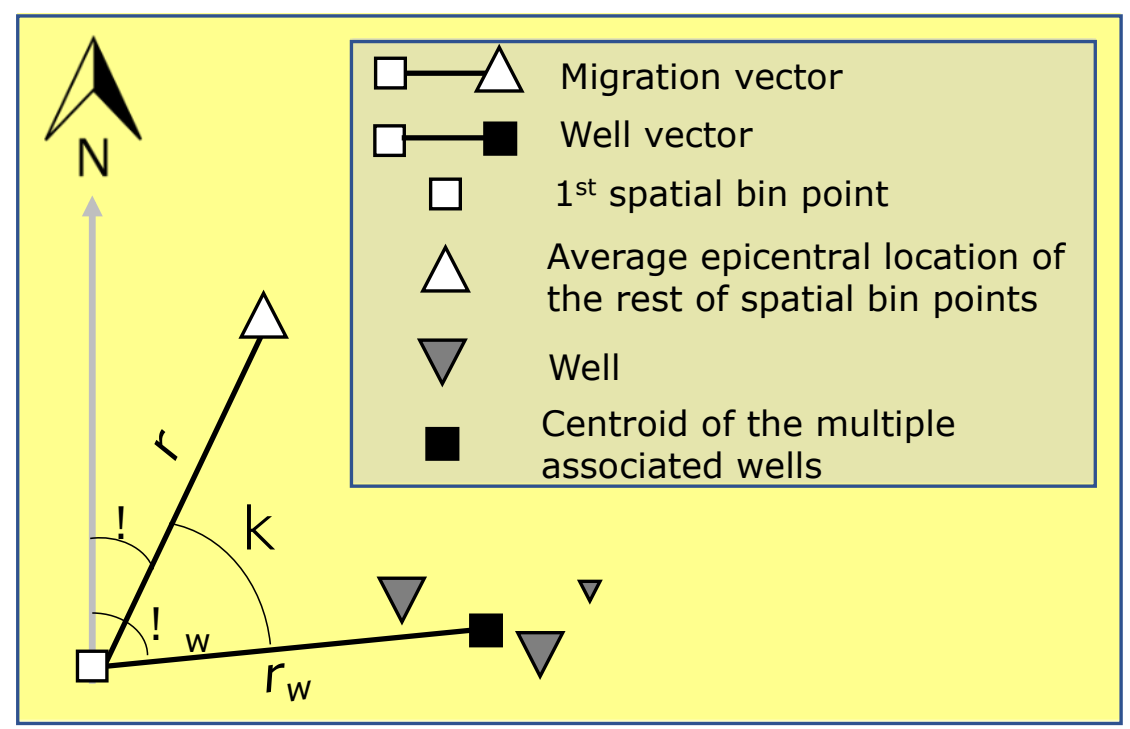

Figure 2. Sketch showing the parameters for calculating the migration and well vector for the comprehensive migration analysis with respect to multiple wells. 
(a.1)

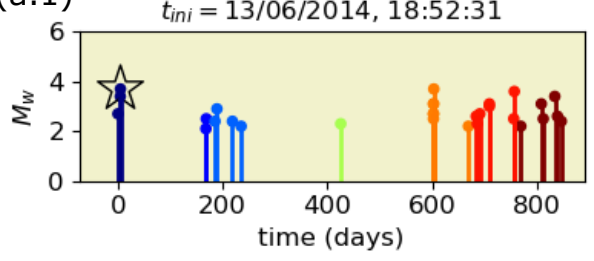

(a.2)

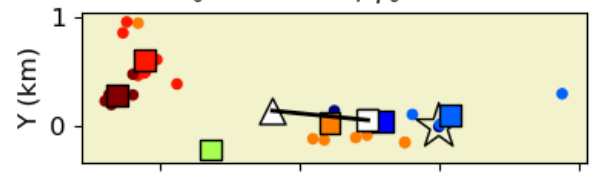

(a.3)

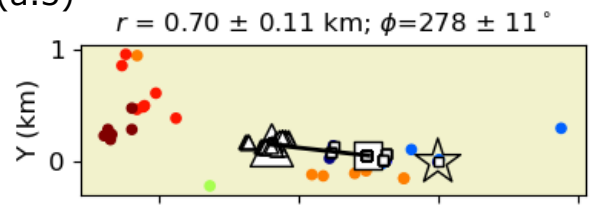

(a.4)

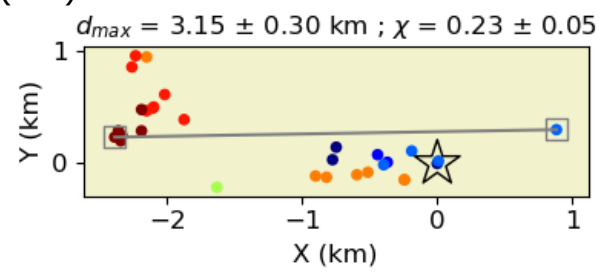

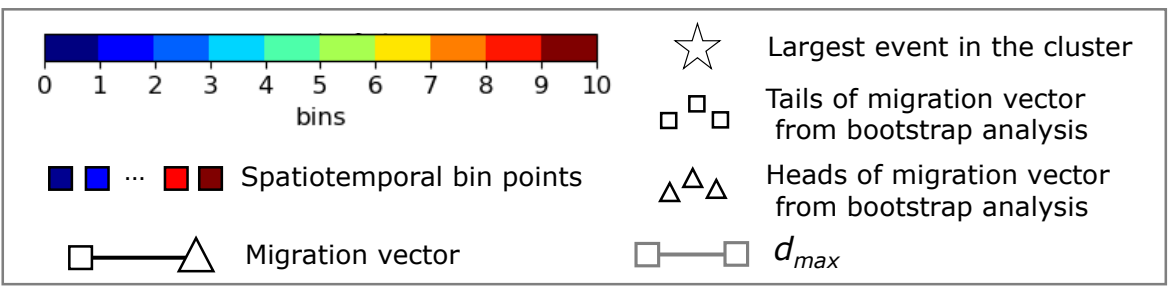

(b.1) $\quad t_{\text {ini }}=07 / 06 / 2014,01: 56: 33$

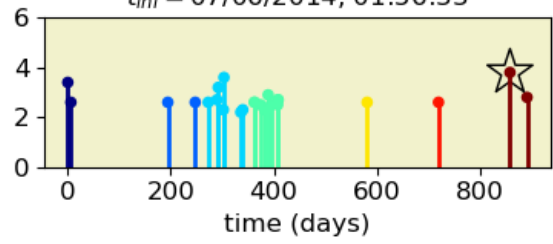

(b.2)

$r_{0}=0.12 \mathrm{~km} ; \phi_{0}=311^{\circ}$

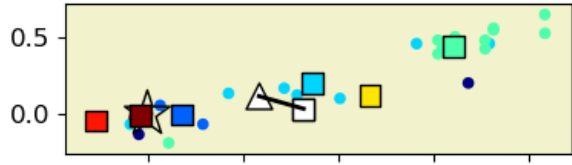

(b.3)

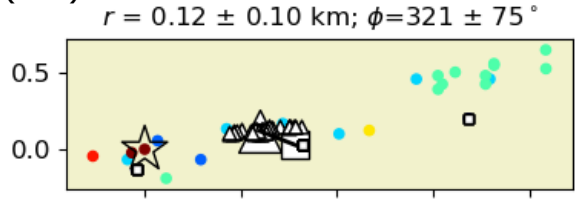

(b.4)

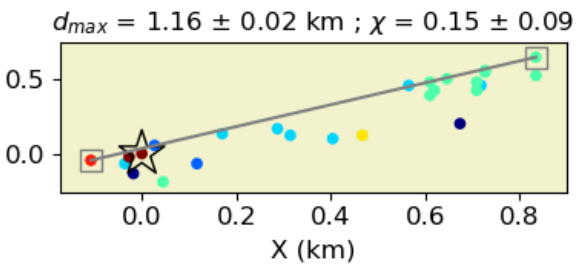

Figure 3. Migration analysis for cluster 52 showing results for a stable migration vector (a) and cluster 17 for an unstable migration vector (b). (a.1, b.1) Temporal evolution of the seismic sequence from tini; the color scale indicates association of seismic events with temporal bins. (a.2, b.2) Migration vector calculation; $r o$ and $\Phi_{0}$ represent the length and azimuth of the migration vector calculated using all events in the cluster. (a.3, b.3) Bootstrap analysis to calculate the final length $r$ and azimuth $\Phi$ of the migration vector and their uncertainties. Small white triangles and small white squares depict the heads and tails of 100 migration vectors for the bootstrap analysis. The final migration vector is depicted by a black line from the tail (large white square) to the head (large white triangle). (a.4, b.4) The maximum cluster length ( $\left.d_{\max }\right)$ and the migration coefficient $(\chi)$ are shown with the uncertainties obtained from the bootstrap analysis. $d_{\max }$ (gray line), is defined by the two seismic events farthest from each other (open gray squares). 


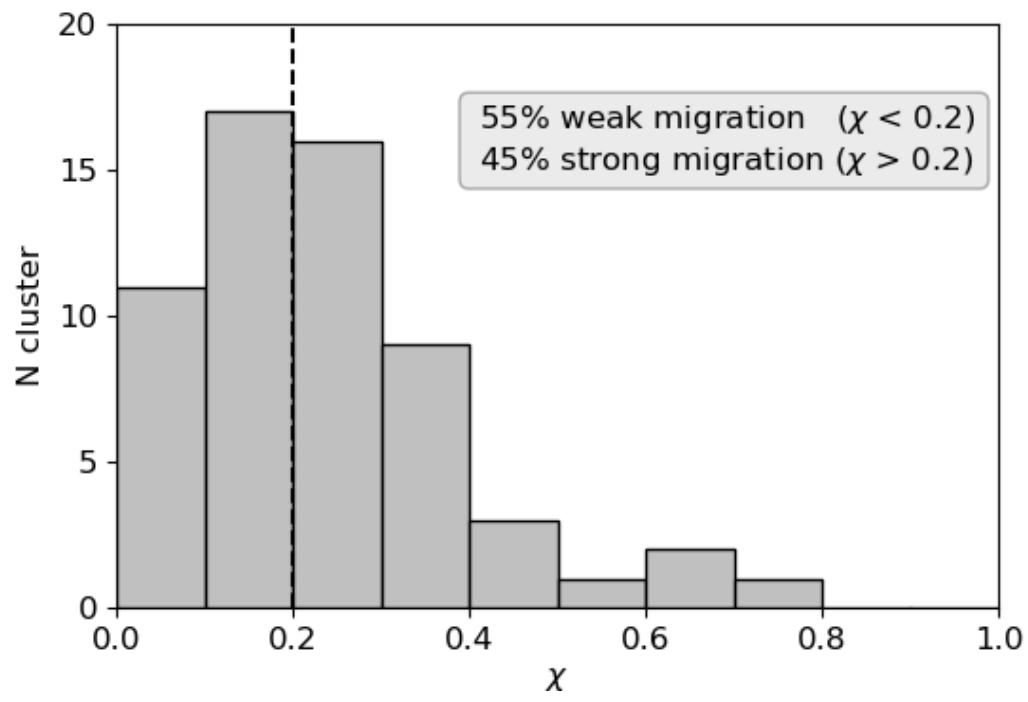

Figure 4. Histogram for the migration coefficient $(\chi)$ for earthquakes clusters in Oklahoma. The dashed black lines separates weak and strong migration clusters. 

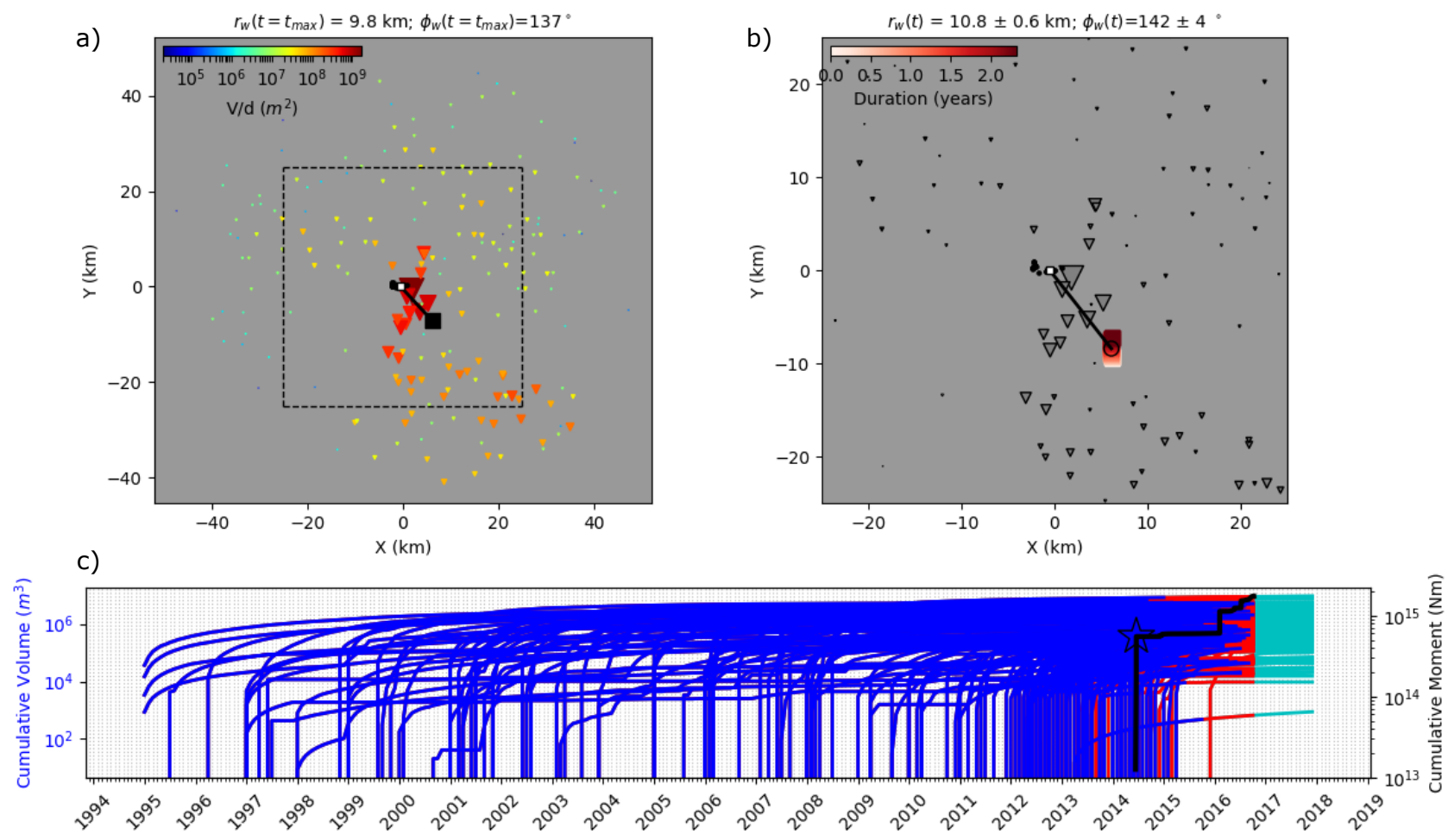

Figure 5. Calculating the well vector for cluster 52 considering the cumulative-injected volume weighting and a diffusion coefficient of $1.5 \mathrm{~m} 2 / \mathrm{s}$. a) Situation at the final time of the seismic sequence tmax. The well vector (black line) is defined from the tail of the migration vector (white square defined in Figure a.3) to the injection midpoint (black square). Length $\left(r_{w}\right)$ and azimuth $\left(\Phi_{\mathrm{w}}\right)$ of the well vector are indicated in the figure header. Wells (inverted triangles) are scaled (color and size) according $\mathrm{V}\left(\mathrm{t}_{\max }-\mathrm{tD}\right) / \mathrm{d}$. b) Location of injection midpoints during the seismic sequence (color-coded squares). The final injection midpoint is shown with an open black circle, the final well vector by the black line. Wells are scaled in size as in a). Only the dashed rectangle from a) is shown. c) Cumulative injected volume for the wells associated with the cluster (blue lines) and cumulative seismic moment for the seismic sequence (black line; the largest event is indicated by the star). Red lines indicate the volume that did not affect the cluster due to diffusion constraints, cyan lines indicate data available after the end of seismic sequence. 


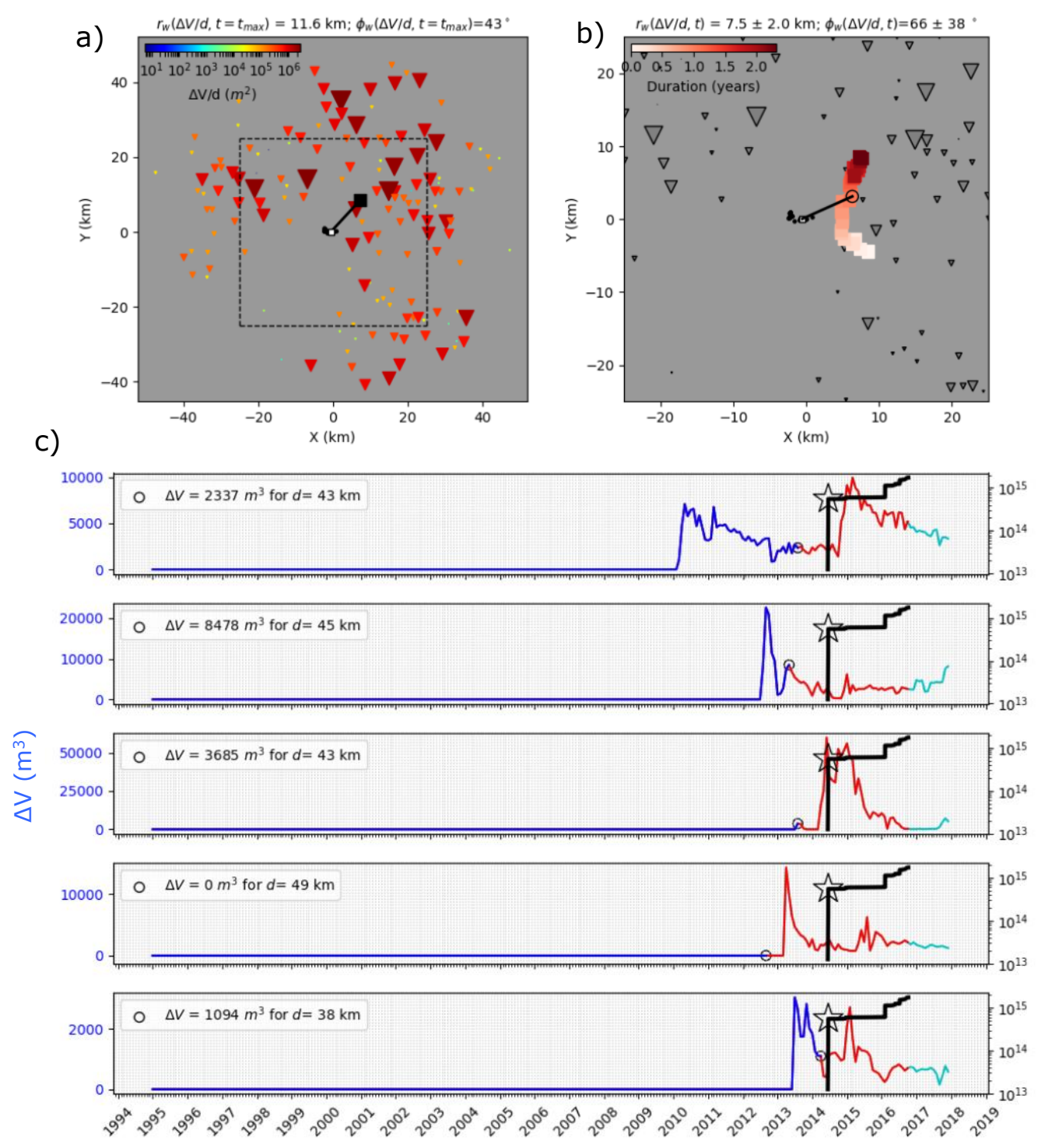

Figure 6. Calculating the well vector for cluster 52 considering the injection-rate volume weighting and a diffusion coefficient of $1.5 \mathrm{~m} 2 / \mathrm{s}$. a) Situation at the final time of the seismic sequence tmax. The well vector (black line) is defined from the tail of the migration vector (white square defined in Figure a.3) to the injection midpoint (black square). Length $\left(r_{w}\right)$ and azimuth $\left(\Phi_{\mathrm{w}}\right)$ of the well vector are indicated in the figure header. Wells (inverted triangles) are scaled (color and size) according $\Delta \mathrm{V}\left(\mathrm{t}_{\max }-\mathrm{t} \mathrm{D}\right) / \mathrm{d}$. b) Location of injection midpoints during the seismic sequence (color-coded squares). The final injection midpoint is shown with an open black circle, the final well vector by the black line. Wells are scaled in size as in a). Only the dashed rectangle from a) is shown. c) Injection rate volumes for five wells associated with the cluster (blue lines) and cumulative seismic moment for the seismic sequence (black line; the largest event is indicated by the star). Red lines indicate the volume that did not affect the cluster due to diffusion constraints, cyan lines indicate data available after the end of seismic sequence. For each well the injection rate volume $(\Delta \mathrm{V})$ with respect to tmax (open black circle) and the well distance (d) are indicated. 

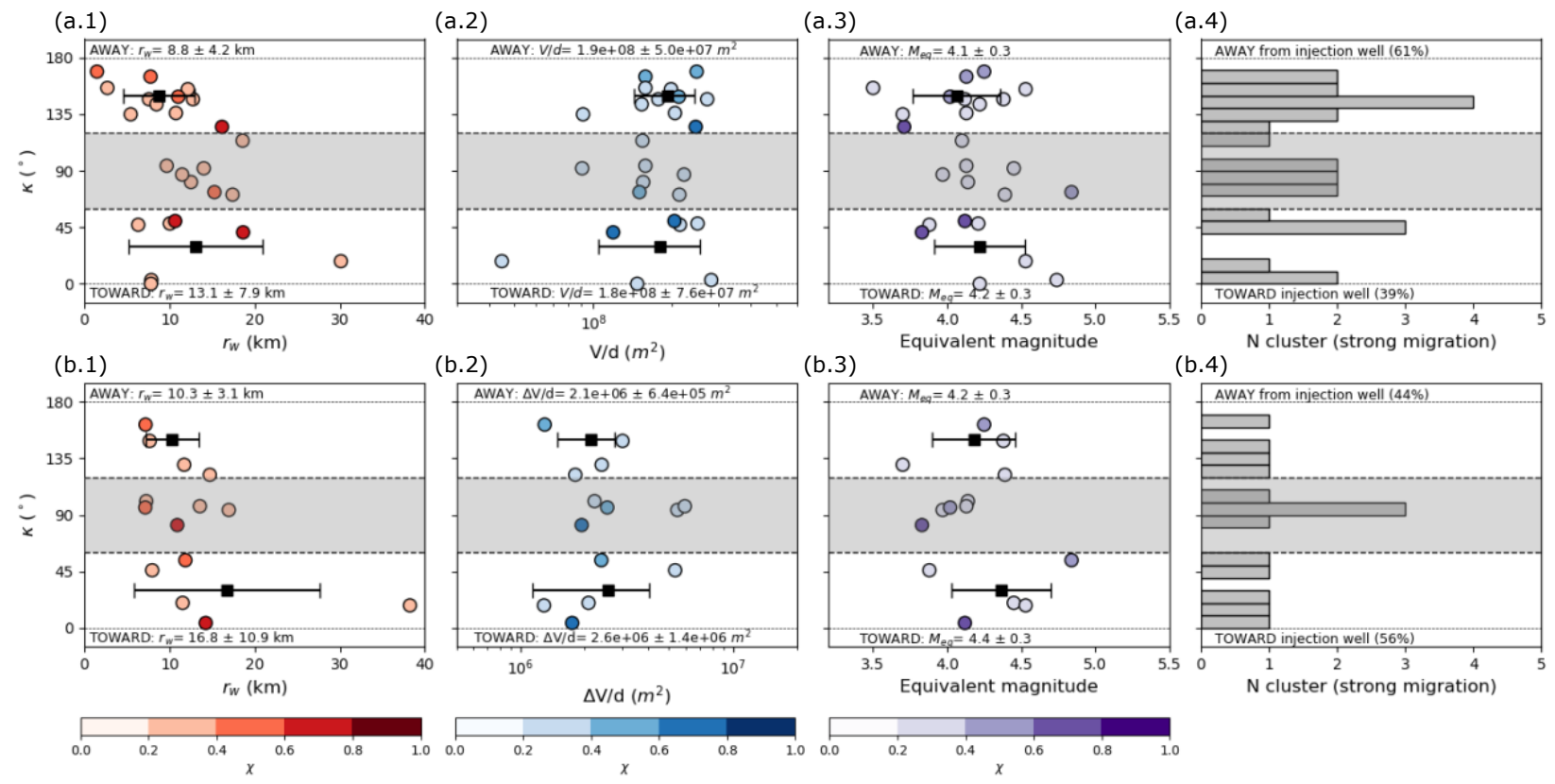
(b.4)

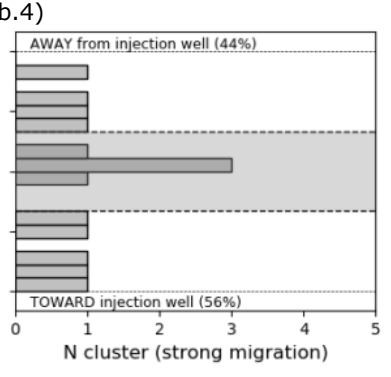

Figure 7. Lateral migration patterns toward or away from injection wells characterized by $\kappa$ values for a diffusion coefficient of $1.5 \mathrm{~m} 2 / \mathrm{s}$. $\kappa$-values for strong migration clusters $(\chi>0.2)$ are plotted as circles scaled in color according the migration coefficient $(\chi)$, considering the cumulative-injected volume weighting (a) and the injection-rate volume weighting (b). Results are shown for each cluster according to the length of the well vector (a.1 and b.1), the total weights assigned to the multiple associated wells in relation to cumulative injected volumes (a.2) and injection rate volumes (b.2) and the equivalent magnitude (a.3 and b.3). Average values and error bars (black squares and lines) are indicated for propagation toward $(\kappa<60 \square)$ and away ( $\kappa$ $>120 \square$ ) from the injection point (see labels). Histograms are also shown including percentages values (a.4 and b.4). Intermediate cases $(60 \square<\kappa<120 \square$ ) are not considered (gray background separated by black dashed lines). 

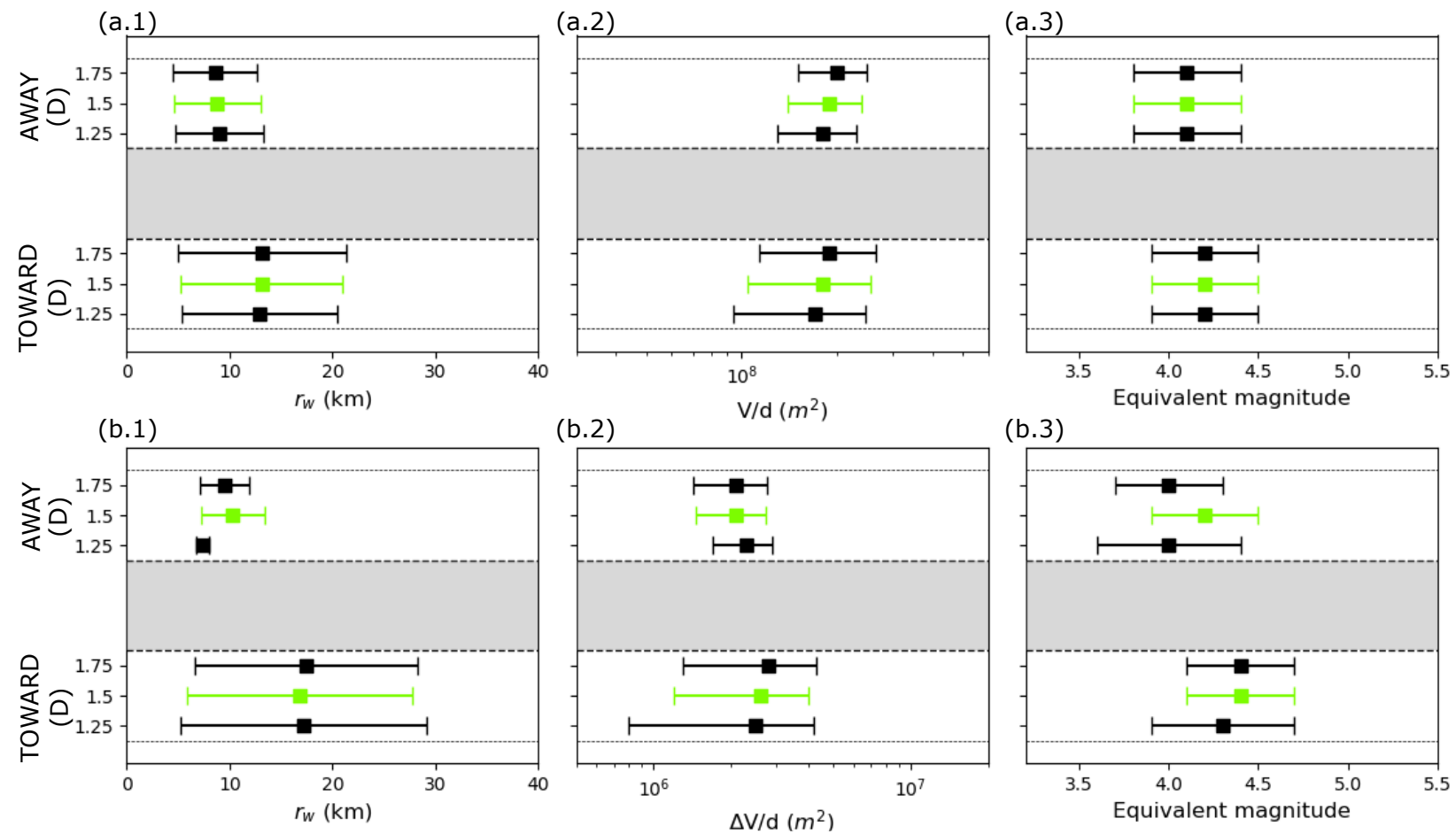

Figure 8. Uncertainties of lateral migration patterns toward or away from injection wells assessed for different diffusion coefficients (D) indicated in the y-axis, considering the cumulative-injected volume weighting (a) and the injection-rate volume weighting (b). Results are shown according to the length of the well vector (a.1 and b.1), the total weights assigned to the multiple associated wells in relation to cumulative injected volumes (a.2) and injection rate volumes (b.2), and the equivalent magnitude (a.3 and b.3). A gray band, as in Figure 7, is shown in each plot to separate both resulting sets (toward and away). Results from Figure 7 for $D=1.5$ $\mathrm{m} 2 / \mathrm{s}$ are indicated in green color. 